\title{
LA DINÁMICA ESPACIOTEMPORAL DE UNA POBLACIÓN MULTIESPECÍFICA DE LOMBRICES EN UN RODAL DE ROBLE
}

\author{
Fernando R. Momo ${ }^{1} \&$ Liliana B. FALCo ${ }^{2}$ \\ ${ }^{1}$ Universidad Nacional de General Sarmiento, Instituto de Ciencias, J.M Gutiérrez 1150, 1613, Los \\ Polvorines, Argentina. E-mail: fmomo@ungs.edu.ar. \\ ${ }^{2}$ Universidad Nacional de Luján, Departamento de Ciencias Básicas, CC 221, 6700, Luján, Argentina.
}

Momo, F. R. \& L. B. Falco. 2010. La dinámica espaciotemporal de una población multiespecífica de lombrices en un rodal de roble. Acta Zoológica Mexicana (n.s.), Número Especial 2: 203-210.

RESUMEN. Se estudió la dinámica de una población multiespecífica de lombrices endogeas en un rodal de robles (Quercus robur) tomando muestras en pequeños parches sobre un mosaico. Se tomaron muestras con reposición en una grilla de 30 puntos equidistantes. La frecuencia de muestreo fue estacional (cada tres meses) durante cinco estaciones. A partir de los datos de abundancias se estimaron las tasas de crecimiento en cada punto y se correlacionaron con las abundancias en el mismo punto y en los puntos vecinos para detectar la dirección de máxima correlación que resultó ser NO-SE. La velocidad migratoria media de las lombrices en esa dirección fue de $1.2 \mathrm{~m} \mathrm{mes}^{-1}$. Se pusieron a prueba cuatro modelos de dinámica de abundancias; dos de ellos, el modelo logístico de Smith (que incorpora los diferentes requerimientos energéticos según el tamaño individual) y la logística asimétrica (que considera competencia intraespecífica con variación no lineal con la densidad) mostraron un ajuste significativo a los datos, revelando un predominio de competencia difusa no jerárquica, una baja tasa de consumo de recursos por individuo y una tasa de crecimiento muy alta a densidades pequeñas y casi constante a densidades por encima de los 20 individuos $\mathrm{m}^{-2}$.

Palabras clave: Oligochaeta, dinámica espacial, correlación, modelos de crecimiento.

Momo, F. R. \& L. B. Falco. 2010. Spatio-temporal dynamics of a multi-specific earthworm population in an oak woodland. Acta Zoológica Mexicana (n.s.), Número Especial 2: 203-210.

ABSTRACT. We studied the spatial dynamics of endogeic earthworms in a Quercus robur forest. Samples were taken using a 30 point grid every three months during a year plus a summer. Growth rates were estimated for each point based on abundance data. Abundances appeared to be correlated in the direction NW-SE showing a net movement of the organisms to the SE region of the studied field. The migration speed of earthworms was $1.2 \mathrm{~m} \mathrm{month}^{-1}$. We fitted the spatial dynamics to several growth mathematical models; the most accurate models were the logistic model of Smith, that is a model with physiological constraints, and the non-symmetric logistic equation, that considers inter-specific competition. The models revealed a significant non-hierarchic diffuse competition and a low rate of individual resource consumption. Moreover, growth rates were high at low population densities but remained roughly constant at densities above 20 individuals $\mathrm{m}^{-2}$.

Keywords: Oligochaetes, spatial and temporal dynamics, correlation, growth models.

Recibido: 16/05/2008; aceptado: 08/01/2010. 


\section{INTRODUCCIÓN}

El estudio ecológico de las lombrices de tierra reviste especial importancia por tratarse de organismos que pueden producir cambios importantes en la estructura del suelo y a la vez actuar como indicadores biológicos de las condiciones del ecosistema edáfico (Lobry de Bruyn 1997, Knoepp 2000, Momo et al. 2003, Römbke et al. 2005).

Por otra parte, las comunidades y poblaciones de lombrices se pueden muestrear mediante métodos estandarizados de dificultad relativamente baja (Anderson \& Ingram 1993), observar e identificar sin mayores problemas, al menos en el caso de la zona de estudio y, por lo tanto, pueden utilizarse para estudios de dinámica que sirven para contrastar hipótesis ecológicas y modelos.

Buscando determinar el modelo de dinámica y velocidad de dispersión de las lombrices de tierra, estudiamos la dinámica de la población total de lombrices endogeas, es decir lombrices que habitan el interior del suelo alimentándose de tierra y realizando galerías (Bouché 1977). El estudio se hizo en un rodal de roble de 52 años de antigüedad (descrito en el trabajo de Falco et al. 1995) y se consideró la totalidad de las lombrices como una población, dado que todas las especies endogeas del sitio tienen requerimientos y tamaños similares.

\section{MATERIAL Y MÉTODOS}

El estudio se realizó en un campo ubicado en el N-E de la provincia de Buenos Aires, Argentina (Fig. 1). Este campo tiene un suelo Argiudol típico (Sfeir et al. 1988) y alberga una comunidad de lombrices de tierra constituida por 15 especies (Falco et al. 1995); en el caso de este trabajo, se consideró la población pluriespecífica formada

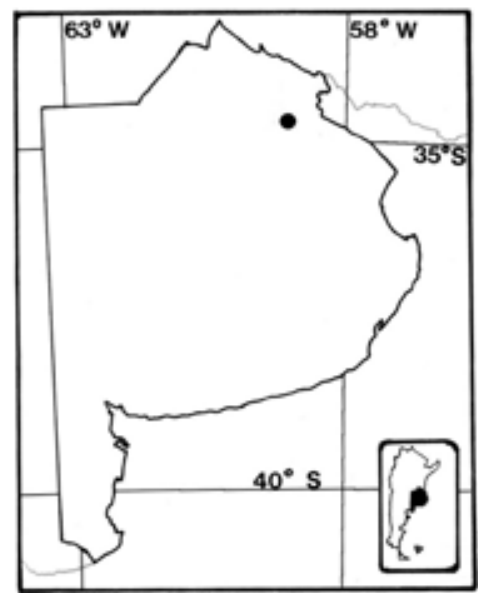

Figura 1. Ubicación del campo donde se realizó el estudio dentro de la provincia de Buenos Aires. El recuadro pequeño muestra la situación de la provincia de Buenos Aires dentro de Argentina. 
por las lombrices del género Aporrectodea (Familia Lumbricidae), que en nuestro caso abarcó tres especies: A. caliginosa, A. trapezoides y A. rosea. Por tratarse de especies con requerimientos, fisiología y dinámica muy similares, se las trató como una única población desde el punto de vista de la dinámica.

Los Argiudoles típicos son suelos profundos con horizontes superficiales bien provistos de materia orgánica (valores que oscilan alrededor del $5 \%$ ) y de unos $25 \mathrm{~cm}$ de espesor, color muy oscuro, francos, de buena estructura; tienen buen drenaje superficial y profundo y son suelos de alta aptitud agrícola. El campo bajo estudio tiene una forestación de robles (Quercus robur) coetáneos (52 años al comienzo de nuestro muestreo) equidistantes unos $8 \mathrm{~m}$ entre árboles; se trata de una especie exótica en la región ya que la vegetación originaria de la zona pampeana es de tipo herbácea y los robles, de origen europeo, fueron introducidos en toda esta zona a partir del año 1890, de la mano con la expansión de la frontera ganadera (Cabrera \& Willink 1973).

Se tomaron muestras con reposición en pequeños parches sobre un mosaico, en una grilla regular de 30 puntos, obteniendo volúmenes de $48 \mathrm{dm}^{3}$ de suelo por muestra, los cuáles se examinaron a mano contando los ejemplares de lombrices. La frecuencia de muestreo fue estacional (cada tres meses) durante cinco estaciones (el primer y último muestreo se realizaron en sucesivos veranos), en los años de 1992 y 1993. A partir de los datos de abundancias se estimaron las tasas de crecimiento en cada punto y se correlacionaron con las abundancias en el mismo punto y en los puntos vecinos para detectar la existencia o no de una dirección de máxima correlación que podría estar indicando una dirección preferencial de migración. Se realizaron mapas de isolíneas de densidad mediante el método de interpolación por Kriging con un ajuste exponencial de los semivariogramas; para esto se utilizó el software GS+ ${ }^{\circledR}$ de Gamma Design Software.

Una vez establecida la dirección de migración se pusieron a prueba cuatro modelos de dinámica de abundancias (Hutchinson 1981): el modelo logístico clásico, el modelo de logística asimétrica, el modelo logístico de Smith (que incorpora los diferentes requerimientos energéticos según el tamaño individual) y la logística discreta de Hassell.

\section{Los modelos de crecimiento}

El modelo de crecimiento poblacional más comúnmente usado en ecología es el de Verhulst (Momo \& Capurro 2006), conocido generalmente como modelo "logístico" de crecimiento. En este modelo la tasa de crecimiento per-cápita desciende en forma lineal con la densidad poblacional. La expresión matemática de este fenómeno es $\frac{1}{N_{i}} \frac{\left(N_{f}-N_{i}\right)}{\Delta t}=r-\frac{r}{K} N_{i}$ donde $N_{i}$ y $N_{f}$ son los números inicial y final de individuos respectivamente para el intervalo de tiempo elegido, $r$ es la tasa de crecimiento máxima y $K$ es la capacidad de carga. Este modelo de crecimiento indica que la población 
crece aceleradamente al principio (hasta alcanzar la mitad de su capacidad de carga) y luego el crecimiento se desacelera, alcanzándose una densidad máxima de individuos $(K)$ de equilibrio.

Otros modelos matemáticos predicen un comportamiento similar en cuanto a la existencia de una densidad máxima de equilibrio pero con diferentes comportamientos en cuanto a la relación entre tasa de crecimiento y densidad. Hay dos que son especialmente interesantes en este contexto: la logística asimétrica (Gilpin \& Ayala 1973) que permite simular varias situaciones, y la ecuación logística de Smith (Smith 1963) que representa un modelo donde el consumo de recursos de los individuos varía con su tamaño y/o edad y con la densidad poblacional.

La logística asimétrica se expresa como $\frac{1}{N_{i}} \frac{\left(N_{f}-N_{i}\right)}{\Delta t}=r\left(1-\left(\frac{N_{i}}{K}\right)^{\theta}\right)$. La fórmula es similar a la del modelo de Verhulst pero el exponente $\theta$ introduce una asimetría en el punto de máxima velocidad de crecimiento que en la logística de Verhulst se da cuando la densidad es igual a $K / 2$. Un $\theta>1$ desplaza el punto máximo a la derecha y un valor entre 0 y 1 lo desplaza hacia la izquierda. Este modelo de crecimiento sugiere que el efecto de freno sobre el crecimiento que produce la densidad poblacional puede ser muy alto a bajas densidades $(\theta<1)$ o recién manifestarse a densidades altas más cercanas a la capacidad de carga $(\theta>1)$.

El modelo logístico de Smith introduce una curva cóncava hacia arriba en la relación entre la tasa de crecimiento y la densidad poblacional. El fundamento biológico de la ecuación de Smith es que los animales considerados utilizan diferentes cantidades de alimento según su edad y biomasa. De tal modo que la ecuación de la tasa de crecimiento queda expresada como $\frac{1}{N_{i}} \frac{\left(N_{f}-N_{i}\right)}{\Delta t}=r\left(\frac{K-N_{j}}{K+(r / c) N_{i}}\right)$, donde el parámetro $c$ expresa una tasa combinada de renovación y mantenimiento de los individuos que depende de su edad y/o biomasa.

El último modelo ensayado es un modelo discreto, expresado por una ecuación en diferencias en vez de una ecuación diferencial. En este caso elegimos el modelo de Hassell (Hassell 1988) porque contiene un parámetro que expresa el predominio de la competencia difusa o jerárquica. El modelo se expresa como $N_{t+1}=\lambda N_{t}\left(1+a N_{t}\right)^{-b} \mathrm{y}$ cuando es el valor del parámetro $b$ es mayor que 1, predomina la competencia difusa.

\section{RESULTADOS}

La correlación espaciotemporal de las densidades resultó ser máxima en el sentido NO-SE, mostrando que las lombrices, en la zona bajo estudio, se desplazaban hacia el sudeste y, aunque sus velocidades de dispersión eran bajas, no eran despreciables ya que la velocidad promedio de movimiento observada fue de $1.2 \pm 0.2 \mathrm{~m} \mathrm{mes}^{-1}$. 
En la Fig. 2a-e se puede observar las variaciones de densidad espacial en los cinco momentos de muestreo.
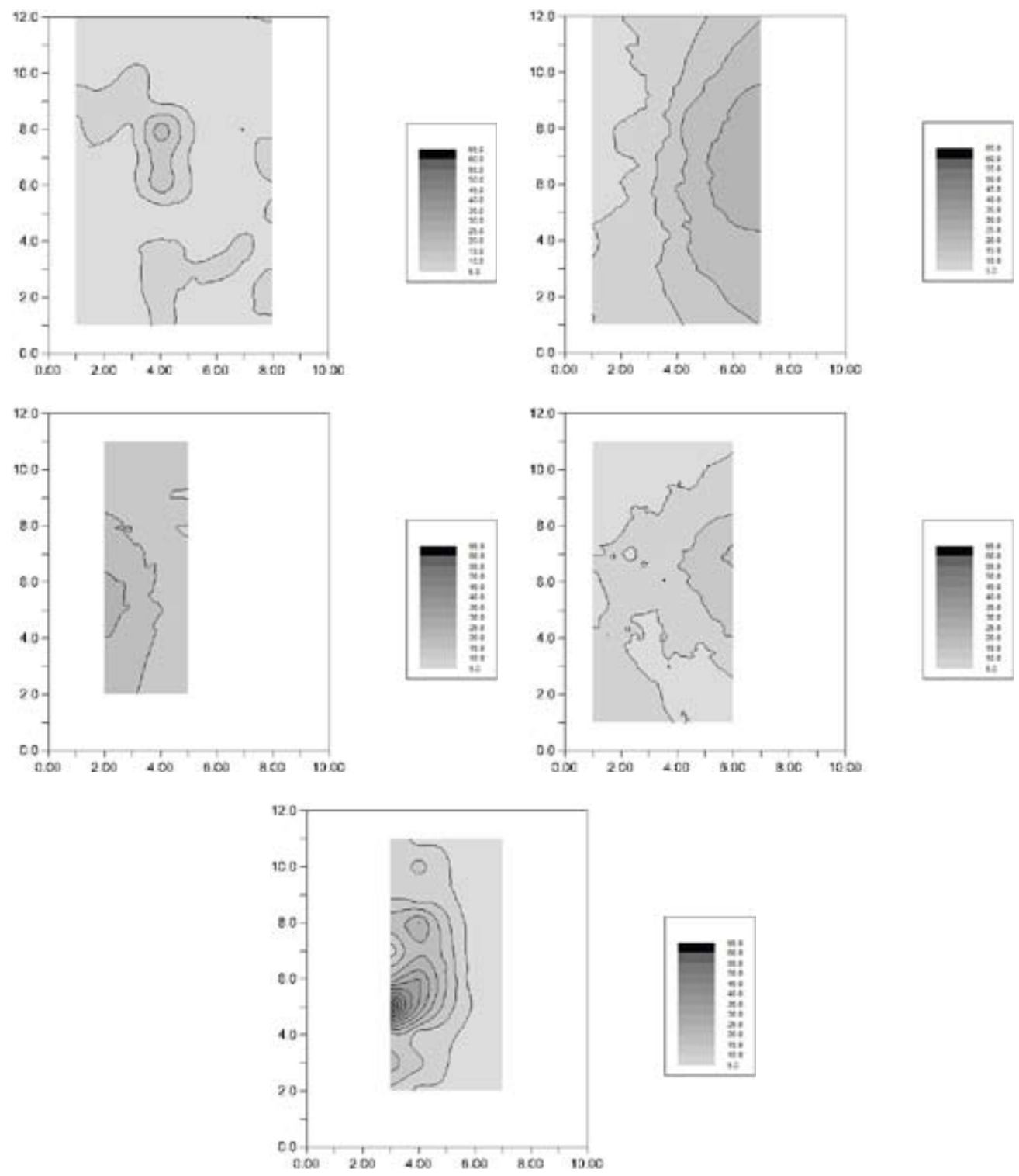

Figura 2. Cambios espaciales en las densidades de lombrices en el campo estudiado durante el año. Las líneas se generaron por krigeo de puntos de isodensidad. (a) verano, 1992; (b) otoño, 1992; (c) invierno, 1992; (d) primavera, 1992; (e) verano, 1993. 
Las tasas de crecimiento por parche se graficaron en función de la suma de los números iniciales en el mismo parche y en su vecino NO. De esta forma se introduce en la dinámica la influencia espacial. Los ajustes a los cuatro modelos se compararon mediante el criterio de información máxima de Akaike (1974) y se observó que los ajustes significativos $(\mathrm{p}<0.05)$ a los datos se produjeron con los modelos de logística asimétrica y de Smith (Fig. 3a y b). Esto indica biológicamente dos cosas: una baja tasa de consumo de recursos por individuo y una competencia difusa y no jerárquica entre ellos que se manifiesta a bajas densidades.
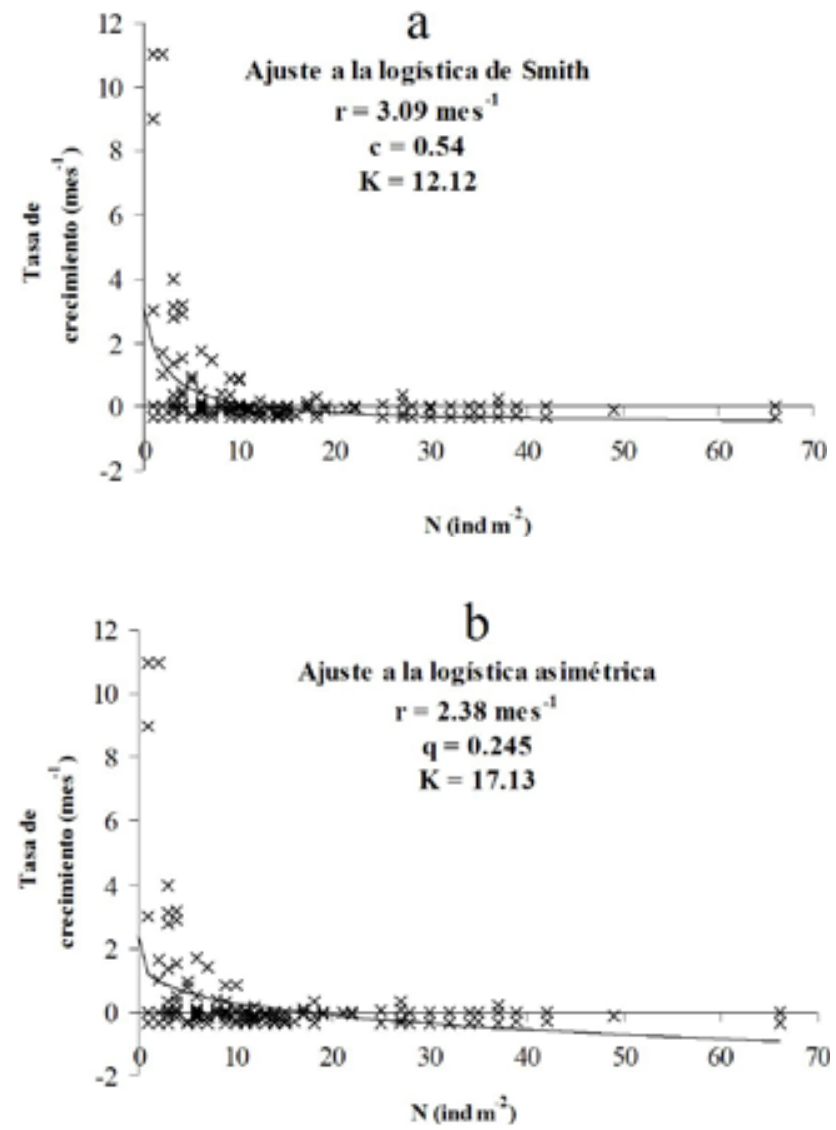

Figura 3. Tasas de crecimiento versus densidades con el ajuste de los datos a la logística de Smith (a) y a la logística asimétrica (b). Las equis representan los datos y las líneas continuas los modelos con los parámetros de ajuste que figuran en cada gráfica. La explicación de los parámetros es la siguiente (ver texto): $r=$ tasa intrínseca de crecimiento de la población, $c=$ tasa de renovación promedio de los individuos, $\theta=$ coeficiente de asimetría de la logística y $K=$ capacidad de carga. 
También se puede observar que para densidades bajas (hasta 20 individuos $\mathrm{m}^{-2}$ ) la tasa de crecimiento per cápita desciende rápidamente; una vez superada esta densidad, la tasa permanece casi constante y cercana a cero (Fig. 3 a y b).

\section{DISCUSIÓN}

Como ha señalado Rossi (2003a, b) la distribución espacial de las lombrices muestra en líneas generales un patrón agregado, con parches de alta concentración separados por claros o zonas de baja concentración numérica o de ausencia de lombrices. Este tipo de patrón sería más común en lombrices de tamaño pequeño y fecundidad alta. Aún a escalas geográficas continentales, algunos autores sostienen que los factores climáticos, las características de los ciclos de vida, la disponibilidad de hábitats y los patrones de actividad humana determinan la dispersión y los patrones espaciales de distribución de las lombrices (Tiunov et al. 2006).

Nuestro trabajo se desarrolla en un área mucho más pequeña donde evidentemente operan mecanismos locales de dispersión a pequeñas distancias y corto plazo. Sin embargo, las variaciones de los patrones espaciales de una época del año a otra sugieren una movilidad importante de las lombrices estudiadas. Esta movilidad puede ser activa y debida específicamente al movimiento de los individuos juveniles o adultos o estar ligada también al movimiento de los cocones que, al ser depositados en superficie, suelen ser arrastrados por el agua durante las lluvias o eventualmente por el viento. En un estudio realizado en una escala muy similar a la nuestra, Hernández et al. (2003) encontraron que los factores que determinan la distribución horizontal de especies endogeas son el contenido de arena gruesa y materia orgánica del suelo y la disponibilidad de nitrógeno; conclusiones similares, en particular con respecto a la textura del suelo y al porcentaje de arena gruesa específicamente fueron observados por Momo et al. (1993) y por Falco et al. (1995). Es posible que esta relación de la abundancia de lombrices con el porcentaje de arena gruesa y de materia orgánica tenga que ver indirectamente con la capacidad de retención de humedad de los suelos (Nordström \& Rundgren 1974, Lee 1985).

Las lombrices estudiadas presentaron un movimiento regular a una velocidad promedio algo por encima de $1 \mathrm{~m}$ por mes. Este movimiento se orientó en una dirección preferencial que se mantuvo a lo largo del año. El resultado final es un patrón espacio-temporal cuya dinámica puede ser descrita por modelos sencillos. Los efectos de competencia intraespecífica se manifestaron a densidades bajas (menores a 20 individuos por metro cuadrado) y la competencia fue difusa.

\section{LITERATURA CITADA}

Akaike, H. 1974. A new look at the statistical model identification. The Institute of Electrical and Electronics Engineers Transactions on Automatic Control. 19: 716-723. 
Anderson, J. M. \& J. S. I. Ingram. 1993. Tropical soil biology and fertility: a handbook of methods, $2^{\text {nd }}$ Ed. CAB International, Wallingford.

Bouché, M. B. 1977. Stratégies lombriciennes. Pp. 122-132. In: U. Lohm and T. Persson (Eds.). Soil organisms as components of ecosystems. Ecological Bulletins, vol. 25, Stockholm.

Cabrera, A. L. \& A. Willink. 1973. Biogeografía de América Latina. Editorial OEA, Washington D.C.

Falco, L. B., Momo, F. R. \& E. B. Craig. 1995. Asociaciones de lombrices de tierra y su relación con la cobertura vegetal en suelos forestados. Revista Chilena de Historia Natural. 68: 523-528.

Gilpin, M. E. \& F. J. Ayala. 1973. Global models of growth and competition. Proceedings of the National Academy of Sciences USA. 70: 3590-3593.

Hassell, M. P. 1988. Dinámica de la competencia y la depredación. Editorial Oikos-Tau, Barcelona.

Hutchinson, G. E. 1981. Introducción a la ecología de poblaciones. Ed. Blume, Barcelona.

Knoepp, J. D., Coleman, D. C., Crsossley Jr., D. A. \& J. S. Clark. 2000. Biological indices of soil quality: an ecosystem case study of their use. Forest Ecology and Management. 138: 357-368.

Lobry de Bruyn, L. A. 1997. The status of soil macrofauna as indicators of soil health to monitor the sustainability of Australian agricultural soils. Ecological Economics. 23: 167-178.

Momo, F. R. \& L. B. Falco. 2003. Meso fauna del suelo. Biología y ecología. Pp.: 51-58. In: A. Albanesi, A. Anriquez, S. Luna, C. Kunst and R. Ledesma (Eds.). Microbiología agrícola. Un aporte de la investigación argentina. Editorial de la Universidad Nacional de Santiago del Estero, Santiago del Estero.

Momo, F. R. \& A. F. Capurro. 2006. Ecología matemática. Principios y aplicaciones. Ediciones Cooperativas, Buenos Aires.

Römbke, J., Jänsch, S. \& W. Didden. 2005. The use of earthworms in ecological soil classification and assessment concepts. Ecotoxicology \& Environmental Safety. 62: 249-265.

Sfeir, A., Costa, M. C., Stavinsky, A., Bonvecchi, V., Penón, E. \& S. Rossi. 1988. Mapa básico de suelos. Universidad Nacional de Luján, Luján.

Smith, F. E. 1963. Population dynamics in Daphnia magna and a new model for population growth. Ecology. 44: 651-653. 